\title{
FORCED OSCILLATIONS WITH RAPIDLY VANISHING NONLINEARITIES
}

\author{
R. KANNAN AND KENT NAGLE
}

(Communicated by Kenneth R. Meyer)

\begin{abstract}
We obtain sufficient conditions for the existence of periodic solutions of nonlinear problems where the nonlinearity vanishes infinitely often.
\end{abstract}

\section{INTRODUCTION}

In this paper we consider the existence of $2 \pi$-periodic solutions of the nonlinear boundary value problem

$$
\begin{gathered}
x^{\prime \prime}+f(x)=p(t), \\
x(0)=x(2 \pi), \quad x^{\prime}(0)=x^{\prime}(2 \pi)
\end{gathered}
$$

where $p(t)$ is $2 \pi$-periodic and $f(x)$ assumes the value zero infinitely often. The case when the distance between successive zeros of $f$ approaches infinity, e.g., $f(x)=\sin (\log x)^{1 / 2}$ for large $x$, was studied in [4], where the terminology of "expansive" nonlinearities for such $f$ was introduced. For additional results and remarks concerning periodic solutions of boundary value problems involving expansive nonlinearities, one is referred to [5, 3, 6]. In [10], this concept was further expanded to the class of $\theta$-expanding nonlinearities.

We present in this paper a unified result connecting the cases when the distances between zeros of $f$ are expansive, equispaced, or shrinking. Our characterization is in terms of the graph of $f$ between two successive zeros and thus is an attempt at deriving a geometric result.

\section{NotATION}

We will assume that (1) can be rewritten in the form

$$
\begin{gathered}
x^{\prime \prime}+g(x) h(x)=p(t), \\
x(0)=x(2 \pi), \quad x^{\prime}(0)=x^{\prime}(2 \pi),
\end{gathered}
$$

Received by the editors July 5, 1989 and, in revised form, November 15, 1989.

1980 Mathematics Subject Classification (1985 Revision). Primary 34B15.

This work was done while the second author was on sabbatical from the University of South Florida, visiting the University of Texas at Arlington. 
where

$p(t)$ is a continuous $2 \pi$-periodic function and $g, h$ are continuous nonnegative real-valued functions.

Let $\mathscr{P}_{2 \pi}$ be the space of continuous $2 \pi$-periodic functions, and for any $y(t) \in$ $\mathscr{P}_{2 \pi}$ we denote by $P$ the linear projection operator defined by

$$
P y(t)=\frac{1}{2 \pi} \int_{0}^{2 \pi} y(t) d t
$$

Then, any $y \in \mathscr{P}_{2 \pi}$ can be expressed uniquely as $y=y_{0}+y_{1}$, where $y_{0}=$ $P y, y_{1}=(I-P) y$, and $\int_{0}^{2 \pi} y_{1}(t) d t=0$.

As in [1], the nonlinear problem (2) is equivalent to the operator equation

$$
x_{1}=H(I-P) N x+P N x \text {, }
$$

where

(i) $N: \mathscr{P}_{2 \pi} \rightarrow \mathscr{P}_{2 \pi}$ is the Nemytskii operator generated by $p(t)-g(x) h(x)$, and

(ii) $H$ is the linear operator defined as follows: $\forall y_{1}(t)$ such that $\int_{0}^{2 \pi} y_{1}(t) d t$ $=0$, let $x_{1}(t)=H y_{1}$ be the unique solution of

$$
\begin{gathered}
x_{1}^{\prime \prime}=y_{1}(t), \quad x_{1}(0)=x_{1}(2 \pi), \\
x_{1}^{\prime}(0)=x_{1}^{\prime}(2 \pi), \quad \text { and } \quad \int_{0}^{2 \pi} x_{1}(t) d t=0 .
\end{gathered}
$$

The operator $H(I-P): \mathscr{P}_{2 \pi} \rightarrow \mathscr{P}_{2 \pi}$ is compact and bounded, and thus corresponding to (3) we have the homotopic problems

$$
x_{1}=\lambda H(I-P) N x+\lambda P N x-(1-\lambda) \varepsilon\left(x_{0}-\bar{x}\right)
$$

where $x=x_{0}+x_{1}$ and $\bar{x}$, a constant, is to be specified later.

Note that (4) is equivalent to the boundary value problem

$$
\begin{gathered}
x^{\prime \prime}=\lambda N x-(1-\lambda) \varepsilon\left(x_{0}-\bar{x}\right)=\lambda\{p(t)-g(x) h(x)\}-(1-\lambda) \varepsilon\left(x_{0}-\bar{x}\right), \\
x(0)=x(2 \pi), \quad x^{\prime}(0)=x^{\prime}(2 \pi) .
\end{gathered}
$$

\section{Estimates}

Before we apply the Leray-Schauder principle to equation (4) or (5), we obtain some preliminary estimates for problem (2). If $z=z_{0}+z_{1}$ is a solution of (2), we have

$$
0=\int_{0}^{2 \pi} z^{\prime \prime}=\int_{0}^{2 \pi}[p(t)-g(z) h(z)] d t .
$$

This implies that

$$
p_{0}=\frac{1}{2 \pi} \int_{0}^{2 \pi} p(t) d t=\frac{1}{2 \pi} \int_{0}^{2 \pi} g(z) h(z) d t
$$


Also

$$
\begin{aligned}
\left|z_{1}^{\prime \prime}\right|=\left|z^{\prime \prime}\right| & =\left|p_{0}+p_{1}(t)-g(z) h(z)\right| \\
& \leq\left|p_{1}(t)\right|+\left|p_{0}\right|+|g(z) h(z)|
\end{aligned}
$$

Hence

$$
\left\|z_{1}^{\prime \prime}\right\|_{L^{1}} \leq\left\|p_{1}\right\|_{L^{1}}+2 \pi\left|p_{0}\right|+\int_{0}^{2 \pi}|g(z) h(z)| d t .
$$

Noting that $g$ and $h$ are nonnegative, we obtain by (6) that

$$
p_{0} \geq 0 \text {. }
$$

Using (6) we find, for any solution $z=z_{0}+z_{1}$ of (2),

$$
\left\|z_{1}^{\prime \prime}\right\|_{L^{1}} \leq\left\|p_{1}\right\|_{L^{1}}+4 \pi p_{0}=\beta
$$

Since $\int_{0}^{2 \pi} z_{1}(t) d t=0$, there exists $t_{0} \in[0,2 \pi]$ such that $z_{1}^{\prime}\left(t_{0}\right)=0$, and hence

$$
\left|z_{1}^{\prime}(t)\right|=\left|\int_{t_{0}}^{t} z_{1}^{\prime \prime}(s) d s\right| \leq\left\|z_{1}^{\prime \prime}\right\|_{L^{1}} \leq \beta .
$$

Therefore,

$$
\left\|z_{1}^{\prime}\right\|_{\infty} \leq \beta
$$

If $z_{1}(t) \not \equiv 0$, there exists a $t_{1}$ such that $z_{1}\left(t_{1}\right)=0$, and thus

$$
\left|z_{1}(t)\right|=\left|\int_{t_{1}}^{t} z_{1}^{\prime}(s) d s\right| \leq 2 \pi\left\|z_{1}^{\prime}\right\|_{\infty} \leq 2 \pi \beta=\gamma .
$$

Thus

$$
\left\|z_{1}\right\|_{\infty} \leq \gamma
$$

Also $\left|z_{1}(t)\right|=\left|z_{1}(t)-z_{1}\left(t_{1}\right)\right| \leq \beta\left|t-t_{1}\right|$, from (9). Thus, for any $\delta>0$, we have

$$
\left|z_{1}(t)\right| \leq \delta, \text { provided }\left|t-t_{1}\right| \leq \delta / \beta
$$

Since $z_{1}$ is periodic, it follows that if

$$
A_{\delta}:=\left\{t \in[0,2 \pi]:\left|z_{1}(t)\right| \leq \delta\right\}
$$

then

$$
\text { meas } A_{\delta} \geq 2 \delta / \beta
$$

We now assume that

$$
\varlimsup_{s \rightarrow-\infty} g(s) h(s)=L
$$

Assumption (H-II) implies that, for any $\alpha>0$ and $\varepsilon>0$, we can find $a<0$ such that

$$
g(x) h(x)<L+\alpha \text { for } x \in[a-\gamma-\varepsilon, a+\gamma+\varepsilon]
$$


and thus

$$
\frac{1}{2 \pi} \int_{0}^{2 \pi} g\left(a+p_{1}(t)\right) h\left(a+p_{1}(t)\right) d t<L+\alpha
$$

for any $\left\|p_{1}\right\|_{\infty}<\gamma+\varepsilon$.

Finally, we assume:

There exist sequences $\left\{a_{k}\right\},\left\{b_{k}\right\}$ such that $\left\{a_{k}\right\}$ is a positive increasing sequence, $\left\{b_{k}\right\}$ is a positive nonincreasing sequence, and

(H-III)

$$
\varliminf_{k \rightarrow \infty} b_{k} c_{k} d_{k}=M, \quad(0<M \leq \infty),
$$

where $c_{k}:=\min g(s)$ and $d_{k}:=\min h(s)$ for $s \in\left[a_{k}-b_{k}\right.$, $\left.a_{k}+b_{k}\right]$.

Using the above notation, we have for any solution $z=z_{0}+z_{1}$,

$$
\begin{aligned}
\frac{1}{2 \pi} \int_{0}^{2 \pi} g\left(a_{k}+z_{1}(t)\right) h\left(a_{k}+z_{1}(t)\right) d t & \geq \frac{1}{2 \pi} \int_{A_{b_{k}}} g\left(a_{k}+z_{1}(t)\right) h\left(a_{k}+z_{1}(t)\right) d t \\
& \geq \frac{1}{2 \pi} c_{k} d_{k} \operatorname{meas}\left(A_{b_{k}}\right) \\
& \geq \frac{b_{k} c_{k} d_{k}}{\pi \beta}, \quad \text { from (12). }
\end{aligned}
$$

Using (H-III), we can conclude that for $\alpha>0$ and $\varepsilon>0$ (we can use the same $\alpha$ and $\varepsilon$ as in (12)) there exists a $b>0$ such that

$$
\frac{1}{2 \pi} \int_{0}^{2 \pi} g\left(b+z_{1}(t)\right) h\left(b+z_{1}(t)\right) d t>\frac{M}{\pi \beta}-\alpha .
$$

When $M=\infty$, we can choose $b$ as large as we want.

\section{Main Result}

Using the $a<0$ and $b>0$ from (12) and (13), we can now define

$$
\begin{aligned}
\Omega=\left\{x(t) \in \mathscr{P}_{2 \pi}: x(t)=x_{0}+x_{1}(t),\left\|x_{1}\right\|_{\infty}<R_{0}, \int_{0}^{2 \pi} x_{1}(t) d t=0,\right. \\
\left.\quad \text { and } a<x_{0}<b\right\}
\end{aligned}
$$

where $R_{0}>\gamma$ (defined in (10)).

In order to discuss the solvability of (2) (or the equivalent operator equation (3)), we apply the Leray-Schauder principle to the homotopic problems defined by (4). We choose $\bar{x}=(a+b) / 2$ in (4), so that when $\lambda=0$, equation (4) becomes linear and has a unique solution $x_{1}=0, x_{0}=(a+b) / 2$, and this is in the interior of $\Omega$.

For any $\lambda \in(0,1)$, note that $(4)$ is equivalent to the differential equation $(5)$. One can repeat the arguments of $\S 3$ that led to the estimate (10) and thereby get 
a bound on $\left|x_{1}(t)\right|$ for any possible solution to (4) uniformly for $\lambda \in(0,1)$. If we choose $R_{0}$ to be larger than this estimate, it follows that (4) does not have a solution on the $x_{1}$-boundary of $\Omega$.

We now show that (4) does not have a solution on the $x_{0}$-boundary of $\Omega$. If possible let there be a solution to (4) when $x_{0}=a$. Then, from (4), we have

$$
\lambda P N x=(1-\lambda) \varepsilon\left(x_{0}-\bar{x}\right)=(1-\lambda) \varepsilon\left(a-\frac{a+b}{2}\right)<0 .
$$

Thus

$$
P N x<0,
$$

or

$$
\frac{1}{2 \pi} \int_{0}^{2 \pi}[p(t)-g(x) h(x)] d t<0 .
$$

This is in contradiction to (13) if

$$
p_{0}=\frac{1}{2 \pi} \int_{0}^{2 \pi} p(t) d t>L,
$$

and thus there is no solution to (4) when $x_{0}=a$. Proceeding similarly, we can show that there is no solution to (4) when $x_{0}=b$ if

$$
p_{0}<M / \pi \beta \text {. }
$$

Hence by the Leray-Schauder principle equation (4) has a solution for $\lambda=1$; that is, (2) has a solution.

Note here that $a$ and $b$ are obtainable in a compatible sense. Given $p_{0}$ and $p_{1}$, we know $\beta$ and hence $\gamma$. Choose $\alpha<M /(\pi \beta)$ and $\varepsilon>0$ arbitrary. Now $a$ and $b$ are chosen so that inequalities (11) and (13) are satisfied.

Summarizing, we have:

Theorem. Under hypotheses (H-I), (H-II), and (H-III), the periodic boundary value problem (2) has at least one solution, provided

$$
L<p_{0}=\frac{1}{2 \pi} \int_{0}^{2 \pi} p(t) d t<\frac{M}{\pi \beta} .
$$

\section{EXAMPLES}

1. Consider the nonlinear problem

$$
\begin{gathered}
x^{\prime \prime}+e^{x}|\sin x|=p(t) \\
x(0)=x(2 \pi), \quad x^{\prime}(0)=x^{\prime}(2 \pi) .
\end{gathered}
$$

In this example, $p(t)$ is $2 \pi$-periodic, $g(x)=e^{x}$, and $h(x)=|\sin x|$, so that the zeros of $f(x)=g(x) h(x)$ are equispaced and are given by

$$
x_{1}=k \pi, k \text { an integer. }
$$

Let $a_{k}=k \pi+\pi / 2$, the midpoint of the two consecutive zeros $k \pi$ and $(k+1) \pi$. Further, let $b_{k}=\pi / 4$, so that $a_{k}-b_{k}$ and $a_{k}+b_{k}$ are both interior to $[k \pi,(k+1) \pi]$. 
Noting that $c_{k}=e^{k \pi+\pi / 4}=\min g(s), d_{k}=\frac{1}{\sqrt{2}}=\min h(s)$, where $s \in$ $\left[a_{k}-b_{k}, a_{k}+b_{k}\right]$, we obtain $\lim _{k \rightarrow \infty} b_{k} c_{k} d_{k}=\infty$. Thus, $M=\infty$, and we also have that $L=0$, where $L$ is as in (H-II). Hence, from the above theorem, problem (15) has a solution provided

$$
0<p_{0}=\frac{1}{2 \pi} \int_{0}^{2 \pi} p(t) d t<\infty .
$$

Finally we note, by integrating (15), that

$$
\frac{1}{2 \pi} \int_{0}^{2 \pi} e^{x}|\sin x| d t=p_{0}
$$

and thus $p_{0} \geq 0$ is a necessary condition for the existence of a solution. The above theorem guarantees the existence of a solution when $p_{0}>0$. When $p_{0}=0$, it follows from (16) that $x=n \pi, n$ an integer. Substituting $x=n \pi$ in (15), we see that $p_{1} \equiv 0$. Hence, when $p_{0}=0, p_{1} \equiv 0$ and the only solutions of $(15)$ are $x=n \pi, n$ an integer.

2. We now consider another example where the zeros of $h(x)$ are equispaced. Thus we consider

$$
\begin{gathered}
x^{\prime \prime}+x^{+}(1+\cos x)=p(t)=p_{0}+p_{1}(t), \\
x(0)=x(2 \pi), \quad x^{\prime}(0)=x^{\prime}(2 \pi),
\end{gathered}
$$

where $x^{+}(t)=\max (0, x(t))$. Thus $f(x)=g(x) h(x)$ has zeros when $x \leq 0$ and when $x=(2 n+1) \pi, n$ a nonnegative integer.

If we proceed as in the previous example, $a_{k}=2 k \pi, b_{k}=\pi / 2, c_{k}=$ $2 k \pi-\pi / 2$, and $d_{k}=1$. Thus $\lim _{k \rightarrow \infty} b_{k} c_{k} d_{k}=\infty$. As in Example 1, we have $L=0, M=\infty$, and, by the theorem, problem (17) has at least one solution if $0<p_{0}<\infty$.

As in Example 1, by integrating (17) we see that a necessary condition for the existence of a $2 \pi$-periodic solution is $p_{0} \geq 0$.

In the case when $p_{0}=0$, integrating (17) gives

$$
\int_{0}^{2 \pi} x^{+}(1+\cos x) d t=0 .
$$

Thus either $x=(2 n+1) \pi$ or $x \leq 0$. If $x=(2 n+1) \pi$, substituting in (17) gives $p_{1}(t) \equiv 0$. But observe that if $y_{1}(t)$ is a solution of

$$
\begin{gathered}
x_{1}^{\prime \prime}=p_{1}(t), \quad x_{1}(0)=x_{1}(2 \pi), \\
x_{1}^{\prime}(0)=x_{1}^{\prime}(2 \pi), \quad \int_{0}^{2 \pi} x_{1}(t)=0
\end{gathered}
$$

then, for any constant $C$ such that $y_{1}(t)+C \leq 0$, it is easy to see that $y_{1}+C$ satisfies

$$
\begin{aligned}
& x^{\prime \prime}+x^{+}(1+\cos x)=p(t)=p_{1}(t), \\
& x(0)=x(2 \pi), \quad x^{\prime}(0)=x^{\prime}(2 \pi) .
\end{aligned}
$$


Thus when $p_{0}=0,(17)$ has infinitely many solutions for any $p_{1}(t)$. 3. Finally we consider the problem

$$
\begin{gathered}
x^{\prime \prime}+e^{x}\left|\sin x^{2}\right|=p(t), \\
x(0)=x(2 \pi), \quad x^{\prime}(0)=x^{\prime}(2 \pi) .
\end{gathered}
$$

This problem has a $2 \pi$-periodic solution if $0<p_{0}<\infty$. This is a case where the zeros of the nonlinearity $e^{x}\left|\sin x^{2}\right|$ are getting closer and closer together as $x \rightarrow \infty$.

Here we take

$$
\begin{aligned}
& a_{k}=k \pi+\pi / 2, \\
& b_{k}=\sqrt{k \pi+3 \pi / 4}-\sqrt{k \pi+\pi / 2} \leq \sqrt{k \pi+\pi / 2}-\sqrt{k \pi+\pi / 4}, \\
& c_{k}=\exp \left(a_{k}-b_{k}\right)=\exp (k \pi+\pi / 2-\sqrt{k \pi+3 \pi / 4}+\sqrt{k \pi+\pi / 2}), \text { and } \\
& d_{k}=1 / \sqrt{2} .
\end{aligned}
$$

Since

$$
\begin{aligned}
\lim _{k \rightarrow \infty} b_{k} c_{k} d_{k}= & \lim _{k \rightarrow \infty}(1 / \sqrt{2})(\sqrt{k \pi+3 \pi / 4}-\sqrt{k \pi+\pi / 2}) \\
& \times \exp (k \pi+\pi / 2-\sqrt{k \pi+3 \pi / 4}+\sqrt{k \pi+\pi / 2}) \\
= & +\infty
\end{aligned}
$$

the upper limit $M$ is $+\infty$. The lower limit is $L=\lim _{s \rightarrow-\infty} g(s) h(s)=0$. It now follows from the theorem that (18) has a $2 \pi$-periodic solution, provided $0<p_{0}<\infty$.

\section{REMARKS}

The study of the above examples illustrates the general idea behind studying the existence of solutions of (1) when $f(x)$ has infinitely many zeros. In fact, the quantity $b_{k} c_{k} d_{k}$ may be viewed as the area of a rectangle inscribed under the curve $f(x)$ between successive zeros of $f$. Further, when the zeros of $h(x)$ are getting closer as $x \rightarrow \infty$, the rapid growth of $g(x)$ can be seen to be critical. Finally, we note that there is no assumption here on the boundedness of $g(x) h(x)$. We do, however, require that $g(x) h(x)$ be bounded on one side.

\section{REMARKS ON A RELATED BUT UNBOUNDED PROBLEM}

We conclude this paper with a discussion of the problem

$$
\begin{gathered}
x^{\prime \prime}+x^{2}|\sin x|=p(t), \\
x(0)=x(2 \pi), \quad x^{\prime}(0)=x^{\prime}(2 \pi),
\end{gathered}
$$

where the nonlinearity $x^{2}|\sin x|$ is nonnegative but "unbounded" both as $x \rightarrow$ $\infty$ and as $x \rightarrow-\infty$.

Since $\varlimsup_{s \rightarrow \infty} g(s) h(s)=+\infty$, assumption (H-II) is not satisfied. However, one can proceed as in the steps leading to inequality (10) in $\S 3$ and obtain a 
bound on $\left\|x_{1}\right\|_{\infty}$. In particular, if $x=x_{0}+x_{1}$ is a solution to (19), then

$$
\left\|x_{1}\right\|_{\infty}<2 \pi\left(\left\|p_{1}\right\|_{L^{1}}+4 \pi p_{0}\right) .
$$

One can also proceed as in the steps following (H-III) leading to (14) and show that, for $b$ large enough,

$$
\frac{1}{2 \pi} \int_{0}^{2 \pi}\left(b+x_{1}(t)\right)^{2}\left|\sin \left(b+x_{1}(t)\right)\right| d t>p_{0}
$$

for any $\left\|x_{1}\right\|_{\infty}<R_{0}$.

We can now construct the set $\Omega$ as in (14) of $\S 4$ and see that the corresponding operator equation (4) does not have a solution on the $x_{1}$-boundary or on the $b$-boundary of $x_{0}$ for $\lambda \in(0,1)$. However in order to show that there is no solution on the $a$-boundary of $x_{0}$, we need bounds on $p_{0}$. In particular, taking $a=0$, one can show that if $x=x_{1}$ is a solution on the $a$-boundary of $x_{0}$, then

$$
p_{0}-4 \pi^{2}\left(\left\|p_{1}\right\|_{L^{1}}+4 \pi p_{0}\right) \leq 0 .
$$

To reach a contradiction, as we did in proving the theorem, we ask when the left-hand side of (21) is positive. A necessary condition is for

$$
0<p_{0}<\left(64 \pi^{4}\right)^{-1} \text {. }
$$

And, for any $p_{0}$ satisfying (22), we must have

$$
0 \leq\left\|p_{1}\right\|_{L^{1}}<(2 \pi)^{-1} \sqrt{p_{0}}-2 p_{0} .
$$

Hence, if $p_{0}$ satisfies (22) and $p_{1}$ satisfies (23), then one can show that (19) has at least one $2 \pi$-periodic solution.

Finally, as remarked in the earlier examples, if $p_{0}=0$, then $p_{1}(t)$ must be identically zero and the solutions are given by $x(t)=n \pi, n$ an integer.

Remark. We would like to make a few remarks on (19) before we conclude this paper. If we let $x=\varepsilon \sin (n t)$, then $x$ is $2 \pi$-periodic and satisfies (19) for

$$
p(t)=\varepsilon^{2} \sin ^{2}(n t)|\sin (\varepsilon \sin (n t))|-n^{2} \varepsilon \sin (n t) .
$$

Choosing $\varepsilon$ sufficiently small and $n$ sufficiently large, we see that (19) has a solution for $p_{0}$ arbitrarily close to zero and $\left\|p_{1}\right\|_{L^{1}}$ arbitrarily large. In this case, $\|x\|_{\infty}$ is still small.

The question of the existence of solutions of (19) for any $p_{0}>0$ is open. In addition it was proved in [8] that the pendulum-type equation

$$
\begin{aligned}
& x^{\prime \prime}+a \sin x=p_{0}+\lambda p_{1}(t)=p(t), \\
& x(0)=x(2 \pi), \quad x^{\prime}(0)=x^{\prime}(2 \pi),
\end{aligned}
$$

where $p$ is $2 \pi$-periodic, has the following interesting property: (24) has a periodic solution for any $p_{1}(t)$ if $p_{0}=0$ and as $|\lambda| \rightarrow \infty, p_{0} \rightarrow 0$ for "any" $p_{1}(t)$. 
By "any" we mean that the set of $p_{1}(t)$ such that $\int_{0}^{2 \pi} p_{1}(t) d t=0$ for which (24) has periodic solutions and the corresponding $p_{0} \rightarrow 0$ is open and dense in the class of all admissible $p_{1}(t)$. It would be interesting to see if a characterization of $p_{1}(t)$ in terms of $p_{0}$ can be done for problem (19).

\section{REFERENCES}

1. L. Cesari, Functional analysis, nonlinear differential equations and the alternative method, in Nonlinear functional analysis and differential equations (Cesari et al., eds.), Marcel Dekker, New York, 1976, pp. 1-192.

2. L. Cesari and R. Kannan, Qualitative study of a class of nonlinear boundary value problems at resonance, J. Differential Equations 56 (1985), 63-81.

3. P. Drabek, Remarks on multiple periodic solutions of nonlinear ordinary differential equations, Comm. Math. Univ. Carolinae 21 (1980), 155-160.

4. S. Fucik, Solvability of nonlinear equations and boundary value problems, Chapter 24, D. Reidel, Holland, 1980.

5. S. Fucik and M. Krbec, Boundary value problems with bounded nonlinearity and generalized nullspace of linear part, Math. Z. 155 (1977), 129-138.

6. P. Hess, A remark on the preceding paper of Fucik and Krbec, Math. Z. 155 (1977), 139-141.

7. R. Kannan and R. Ortega, Periodic solutions of pendulum-type equations, J. Differential Equations 59 (1985), 123-144.

8. $\ldots$ An asymptotic result in forced oscillations of pendulum-type equations, Appl. Anal. (to appear).

9. J. Mawhin, Periodic oscillations of forced pendulum-type equations, Seminaire de Mathematique, $1^{\prime}$ er Semestre 1982, UCL II-1, II-22.

10. K. Nagle and K. Singkofer, Existence and multipli'ity of solutions to nonlinear differential equations at resonance, J. Math. Anal. Appl. 94 (1 883), 222-236. 76019

Department of Mathematics, University of Texas at Arlington, Arlington, Texas

Department of Mathematics, University of South Florida, Tampa, Florida 33620 